\title{
Blind-Source Separation of Multiple Signal Sources of fMRI Data Sets Using Independent Component Analysis
}

\author{
B. B. Biswal and J. L. Ulmer \\ Biophysics Research Institute, Medical College of Wisconsin, Milwaukee, WI USA
}

Purpose. As we seek to establish the use of fMRI as the method of choice for studying systems-level neuroscience, it is essential that we be able to distinguish the various "signal" sources from the "noise" sources in which they are immersed. Almost all published fMRI studies thus far have been used to localize signal changes related to brain activity but not to determine the source of the individual signals. A better understanding of these signal sources could not only lead to improved reliability and SNR of task-induced signal changes, but could also improve our understanding of the biophysical basis of signal origin. In this study, we apply a blind-source separation technique to determine multiple signal sources in FMRI time-series data sets and compare this analysis method to PCA in the same data sets.

Methods. See Ref. 1 for details of ICA methodology. For a given $N$ number of sources in the form of $S^{T}(t)=\left[s_{1}(t)\right.$ $\left.\mathrm{s}_{2}(\mathrm{t}) \ldots \mathrm{s}_{\mathrm{N}}(\mathrm{t})\right]$, where $\mathrm{t}$ denotes time, assuming a mixing process defined as:

$$
\mathbf{X}(\mathrm{t})=\mathbf{H} * \mathbf{S}(\mathrm{t})
$$

where $\mathbf{H}$ is an unknown matrix referred to as mixing matrix. The vector $\mathbf{X}$ is a linear mix of the original sources $\mathbf{S}$. This process can be derived as an idealized approximations of $\mathrm{N}$ physiological sources such as the task-activation, cardiac and respiration induced signals in each voxel of the brain. Therefore, the goal is to recover the $\mathbf{S}$ vectors, given only the known $\mathbf{X}$ vectors. One way in which this can be achieved is by inverting the mixing process:

$$
\mathrm{Y}(\mathrm{t})=\mathbf{W} * \mathbf{X}(\mathrm{t})
$$

and find a $W=\mathbf{H}^{-1}$, so that $\mathbf{Y}(\mathrm{t})=\mathbf{S}(\mathrm{t})$. Finding this matrix $\mathbf{W}$ is difficult since no prior information is known about the $\mathbf{S}$ and $\mathbf{H}$ matrix. An approach suggested by Comon (1) was to implement the unmixing Eq. [2], and optimize with respect to the unmixing matrix, to minimize the statistical independence between the output. Comon used statistical independence by using up to the fourth-order statistics (cummulants).

Experiments were performed using a 3T/60 Bruker scanner and a home-built 3-axis balanced-torque head gradient coil with an endcapped birdcage RF transmit-receive coil. Four subjects ( 3 males and 1 female between 14-29 years of age) with no known neurological disorders, head trauma, contraindication to MR, or medication were scanned. For functional imaging, several time series of 280 images $(280 \mathrm{sec})$ were obtained from multiple axial slices with a gradient-echo EPI sequence, FOV $=24 \times 24 \mathrm{~cm}$, matrix size $=64 \times 64$, slice thickness $=10 \mathrm{~mm}$ (corresponding to a spatial resolution of $3.75 \times 3.75 \times 10 \mathrm{~mm}$ ), TE $=40 \mathrm{msec}$, and $\mathrm{TR}=1000 \mathrm{msec}$ with flip angle $=81^{\circ}$. In each experiment, in addition to the bilateral finger tapping, $5 \% \mathrm{CO}_{2}$ was added to the breathing gas mixture $100 \mathrm{sec}$ into the 280 -sec paradigm.

Results. An increase in the overall magnitude of the signal intensity was seen in all the time-course pixels corresponding to hypercapnia, in addition to the "ON/OFF" bilateral finger tapping responses. However, the magnitude of the task-induced signal change and the hypercapnia response was not uniform and varied among pixels. The pixel time-courses from PCA and ICA are shown in Fig. 1. It was seen that PCA could not reliably differentiate between the hypercapnic response and task-induced response and both are contained in the first principal component. The third principal component, however, contains a response similar to that of a hypercapnic paradigm. Therefore it appears that PCA cannot separate these components, and further, the same signal source might be present in multiple principal components. In all four subjects it was observed that the ICA could reliably separate the task-induced signal source from the hypercapnic signal source. The first and second ICA components contained the task-induced and hypercapnic signal changes, respectively. In three of the four subjects, the third ICA component had a dominant frequency at around $0.12 \mathrm{~Hz}$, approximating the underlying respiratory rate.
Principal Components
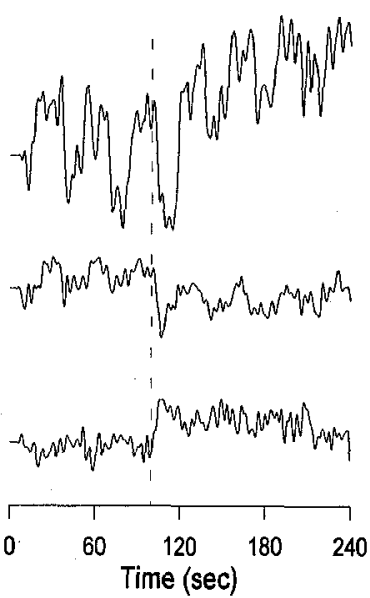

Figure 1. Comparison of ICA and PCA on FMRI data sets.
Independent Components
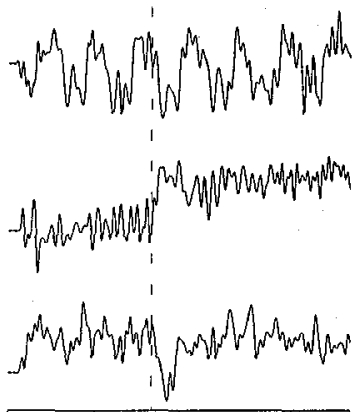

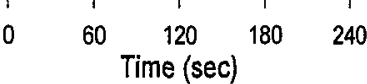

Discussion. This study demonstrates the usefulness of independent component analysis in separating multiple signal sources. It shows that fMRI data sets can be estimated to determine the nature and location of the signal sources. This could not only improve the reliability of fMRI data sets but also enhance our understanding of the signal sources in the human brain. A fundamental limitation of principal component analysis is that it can only separate signals sources that are uncorrelated.

\section{References}

1. Comon, P., Signal Processing 36, 287 (1989).

2. Bell, A.I., Sejnowski, T.J., Neural Computation 7, 1129 (1995). 